\title{
MR Imaging Evaluation of Inferior Olivary Nuclei: Comparison of Postoperative Subjects with and without Posterior Fossa Syndrome
}

\author{
Z. Patay, J. Enterkin, J.H. Harreld, Y. Yuan, U. Löbel, Z. Rumboldt, R. Khan, and F. Boop
}

\begin{abstract}
BACKGROUND AND PURPOSE: Posterior fossa syndrome is a severe postoperative complication occurring in up to $29 \%$ of children undergoing posterior fossa tumor resection; it is most likely caused by bilateral damage to the proximal efferent cerebellar pathways, whose fibers contribute to the Guillain-Mollaret triangle. When the triangle is disrupted, hypertrophic olivary degeneration develops. We hypothesized that MR imaging patterns of inferior olivary nucleus changes reflect patterns of damage to the proximal efferent cerebellar pathways and show association with clinical findings, in particular the presence or absence of posterior fossa syndrome.
\end{abstract}

MATERIALS AND METHODS: We performed blinded, randomized longitudinal MR imaging analyses of the inferior olivary nuclei of 12 children with and 12 without posterior fossa syndrome after surgery for midline intraventricular tumor in the posterior fossa. The Fisher exact test was performed to investigate the association between posterior fossa syndrome and hypertrophic olivary degeneration on MR imaging. The sensitivity and specificity of MR imaging findings of bilateral hypertrophic olivary degeneration for posterior fossa syndrome were measured.

RESULTS: Of the 12 patients with posterior fossa syndrome, 9 had bilateral inferior olivary nucleus abnormalities. The 12 patients without posterior fossa syndrome had either unilateral or no inferior olivary nucleus abnormalities. The association of posterior fossa syndrome and hypertrophic olivary degeneration was statistically significant $(P<.0001)$.

CONCLUSIONS: Hypertrophic olivary degeneration may be a surrogate imaging indicator for damage to the contralateral proximal efferent cerebellar pathway. In the appropriate clinical setting, bilateral hypertrophic olivary degeneration may be a sensitive and specific indicator of posterior fossa syndrome.

ABBREVIATIONS: $\mathrm{HOD}=$ hypertrophic olivary degeneration; $\mathrm{ION}=$ inferior olivary nucleus; $\mathrm{pECP}=$ proximal efferent cerebellar pathway; $\mathrm{PFS}=$ posterior fossa syndrome

$\mathbf{P}$ osterior fossa syndrome (PFS), a complication of posterior fossa surgery, ${ }^{1,2}$ occurs in $11 \%-29 \%$ of patients undergoing posterior fossa tumor resection. ${ }^{3}$ Although the definition of the "all-inclusive" PFS is broad and comprises complex neurobehavioral and motor symptoms, cerebellar mutism is at the core of the diagnosis. ${ }^{4}$

Growing evidence suggests that PFS is the result of bilateral damage to the proximal efferent cerebellar pathways (pECPs)

Received May 15, 2012; accepted after revision August 21.

From the Departments of Radiological Sciences (Z.P., J.H.H., U.L.), Biostatistics (Y.Y.), Pediatrics (R.K.), and Surgery (F.B.), St. Jude Children's Research Hospital, Memphis, Tennessee; and Department of Radiology (J.E., Z.R.), Medical University of South Carolina, Charleston, South Carolina.

This work was supported in part by grant no. CA21765 from the National Cancer Institute and by the American Lebanese Syrian Associated Charities.

Please address correspondence to Zoltan Patay, MD, PhD, Department of Radiological Sciences, St. Jude Children's Research Hospital, 262 Danny Thomas Pl, Mail Stop 210, Memphis, TN 38105; e-mail: zoltan.patay@stjude.org

- Indicates open access to non-subscribers at www.ajnr.org

http://dx.doi.org/10.3174/ajnr.A3762 along the dentatorubrothalamocortical pathway. ${ }^{5-13}$ This relationship was initially observed as cerebellar mutism after stereotactic ablation of the bilateral dentate nuclei. ${ }^{14}$ Reversed cerebellocerebral diaschisis, in which deprivation of the cerebral cortex from cerebellar input due to bilateral pECP damage results in a frontally predominant drop of cerebral cortical perfusion, has been proposed to be the mechanism of PFS, and cerebellar mutism is thought to be a form of speech apraxia. ${ }^{9}$ During the months following surgery, speech and the associated neurologic deficits usually improve, but those rarely if ever completely normalize. This outcome suggests a profound disturbance of complex neural systems, with significant implications for the long-term quality of life of the steadily increasing number of survivors. ${ }^{15}$

Damage anywhere along the dentatorubrothalamocortical pathway may lead to a speech disorder, and damage to the dentate nuclei in particular has repeatedly been cited as a cause of cerebellar mutism, ${ }^{5,10,16,17}$ which can occur after injury along the superior cerebellar peduncles, ${ }^{8}$ brachium pontis/conjunctivum, ${ }^{6,18}$ 
bilateral thalamic tracts, ${ }^{11}$ or the frontal lobes. ${ }^{19-21}$ Given that the dentate nuclei, superior cerebellar peduncles, and mesencephalic tegmental decussation often lie adjacent to and may, therefore, be invaded by midline intraventricular posterior fossa tumors, these structures are the ones most prone to injury during aggressive tumor resection.

The efferent cerebellar tracts that pass through the superior cerebellar peduncles ${ }^{3}$ are adjacent to and involve fibers associated with the dentatorubral segment of the Guillain-Mollaret triangle. ${ }^{22}$ The Guillain-Mollaret triangle is composed of an ipsilateral red nucleus and inferior olivary nucleus (ION), which are connected by the central tegmental tract, and a contralateral dentate nucleus, which is connected through the su-

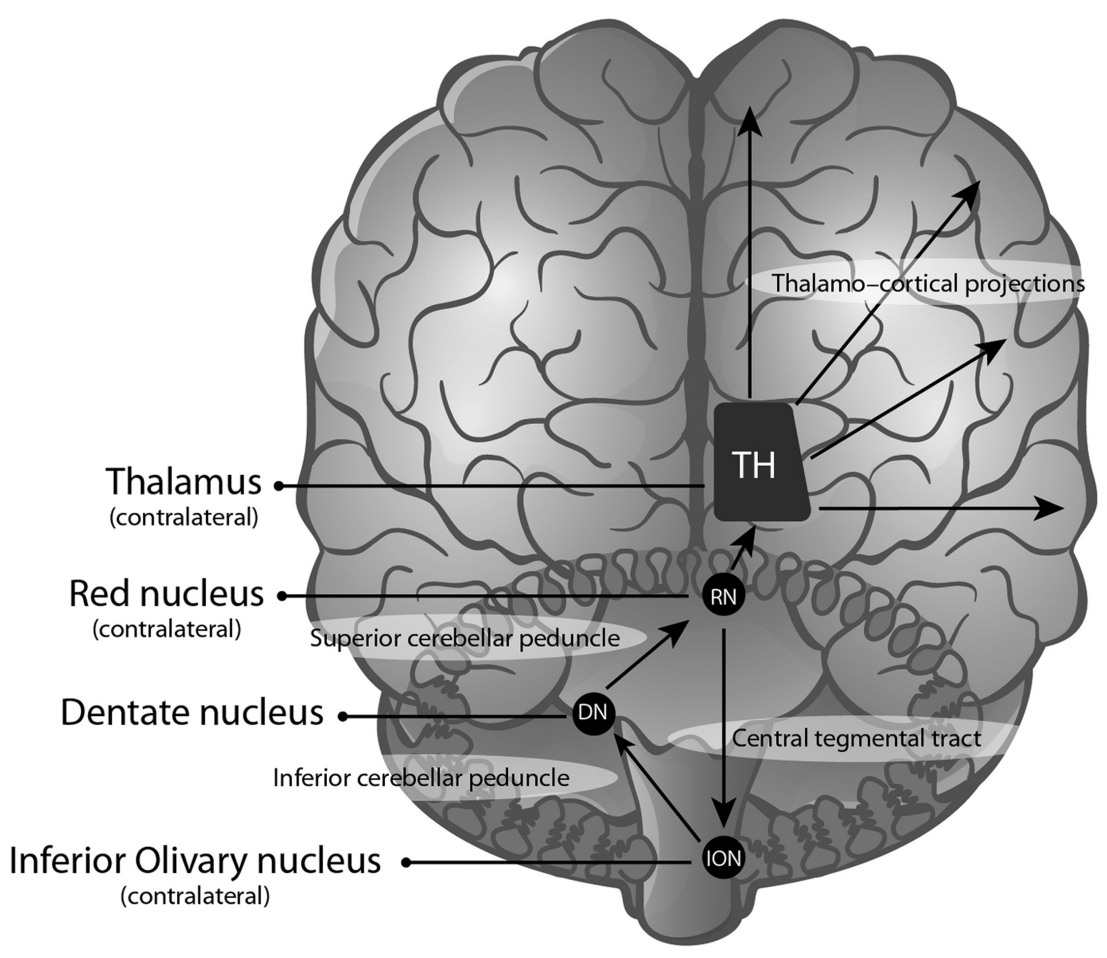

FIG 1. The Guillain-Mollaret triangle and dentatorubrothalamocortical projections.
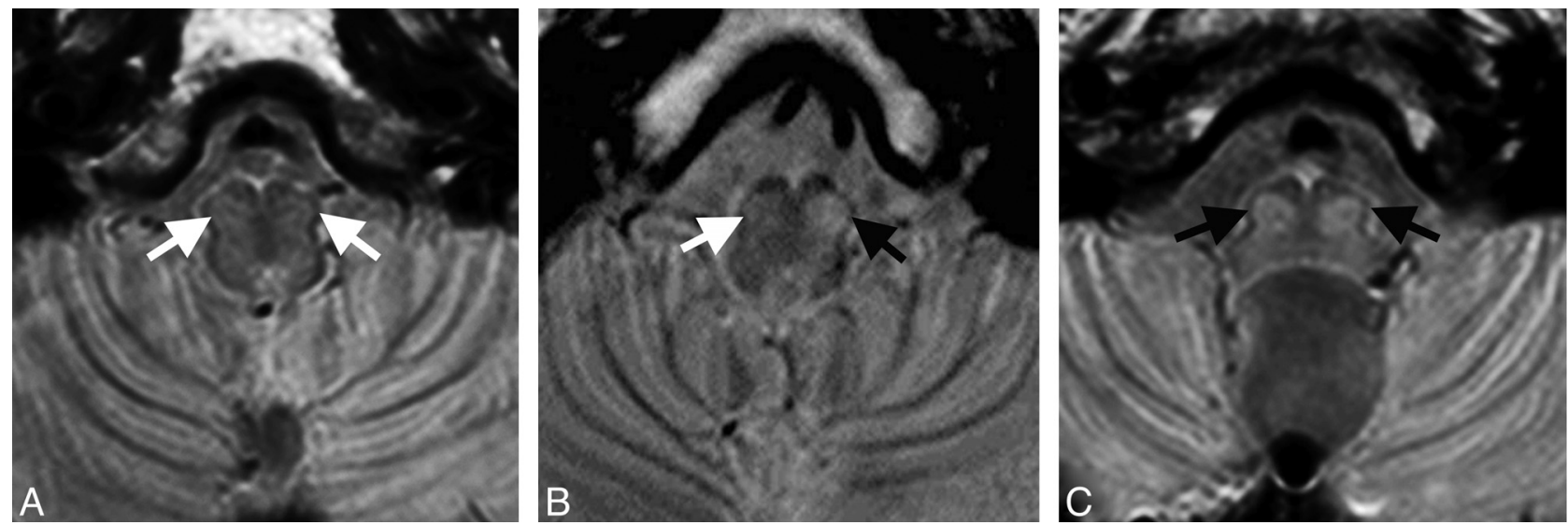

FIG 2. Transverse proton attenuation-weighted images illustrating the MR imaging appearance of normal IONs (A, white arrows) and unilateral (B, black arrow) and bilateral (C, black arrows) HOD in patients approximately 4 months after surgery for midline posterior fossa tumors. Note that with this imaging technique, normal IONs (white arrows) are barely recognizable, but in abnormal conditions, their conspicuity is markedly increased, allowing confident identification of HOD in most cases. 

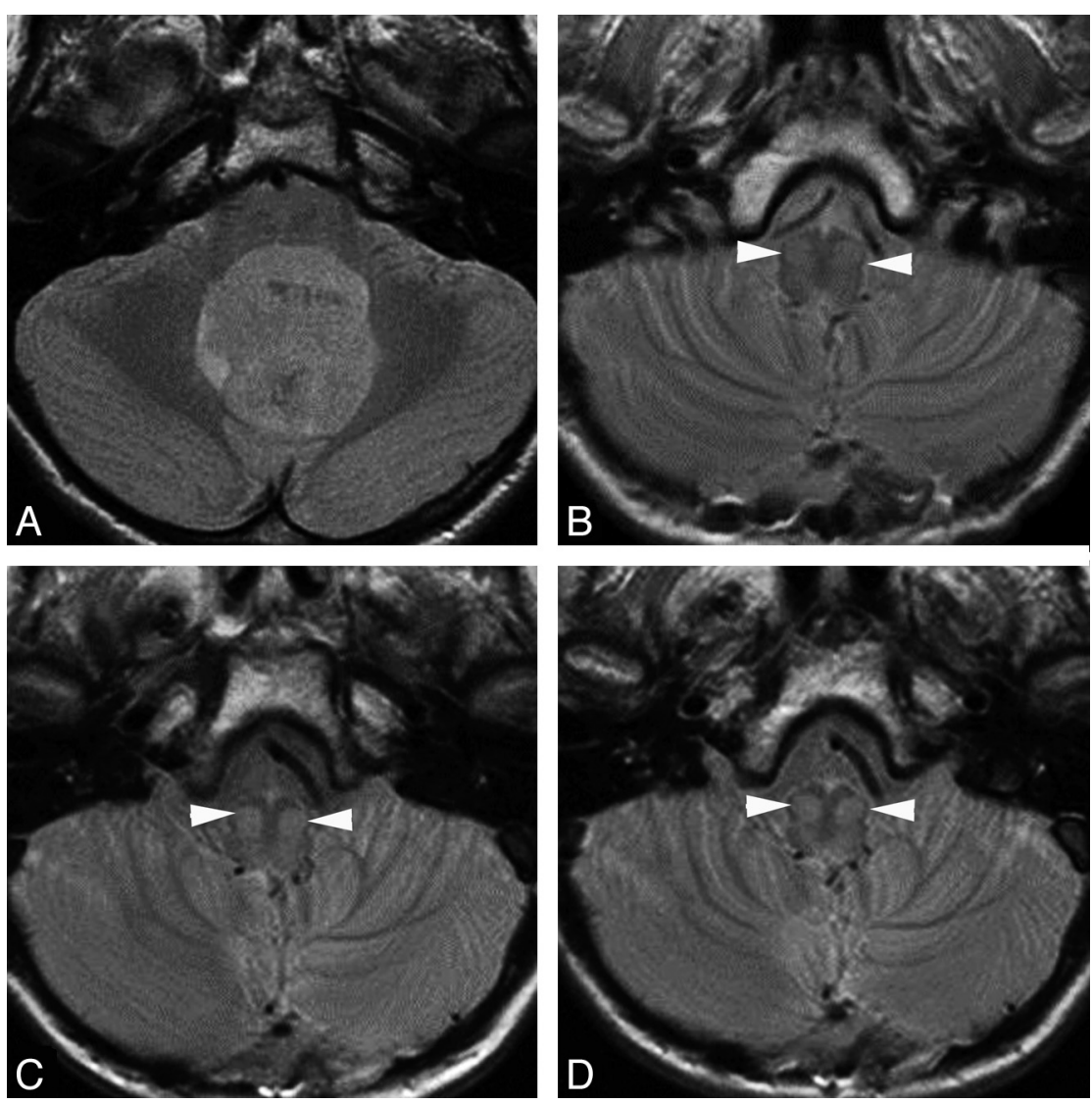

FIG 3. Transverse proton attenuation-weighted images showing chronologic changes (increasing conspicuity) within the bilateral inferior olivary nuclei (white arrowheads) in a patient with PFS during the first year after surgery. A, Preoperative study. B, At 1-month follow-up. C, At 6-month follow-up. D, At 10-month follow-up.

not explained by any other cause such as medication. For comparison, 12 age- and sex-matched patients without PFS were selected as controls. All patients, regardless of clinical PFS status, were evaluated for associated cerebellar signs and symptoms. Of the 12 patients with PFS, 11 had medulloblastoma and 1 had an atypical teratoid/rhabdoid tumor. Of the 12 patients without PFS, all had medulloblastomas.

\section{MR Imaging}

Postoperative follow-up studies included standardized axial long-TR imaging (proton attenuation and $\mathrm{T} 2$-weighted), in addition to multiplanar nonenhanced and contrast-enhanced T1-weighted, axial contrast-enhanced FLAIR, and diffusion-weighted imaging. The geometric parameters for the long-TR imaging sequences (used for evaluation of the ION) were identical: number of sections $=31$, section thickness $=5 \mathrm{~mm}$, FOV read $=210 \mathrm{~mm}$, FOV phase $=$ $100 \%$. Other parameters for the proton attenuation and T2weighted sequence were the following: $\mathrm{TR}=4500 \mathrm{~ms} ; \mathrm{TE} 1=13 \mathrm{~ms}$; $\mathrm{TE} 2=105 \mathrm{~ms}$; turbo factor $=5$; bandwidth $=98 \mathrm{~Hz} / \mathrm{px}$; and for FLAIR: $\mathrm{TR}=10,000 \mathrm{~ms} ; \mathrm{TI}=2500 \mathrm{~ms} ; \mathrm{TE}=103 \mathrm{~ms}$; turbo factor $=$ 21 ; bandwidth $=130 \mathrm{~Hz} / \mathrm{px}$. In compliance with protocol requirements, all studies were performed on 1.5T magnets (Magnetom Avanto; Siemens, Erlangen, Germany) at the same center by using a 4-channel, circularly polarized head array coil. Average follow-up was $38 \pm 20$ months, and each patient had an average of $14 \pm 5$ follow-up studies.

\section{Image Analysis}

Blinded, randomized analysis of MR images was performed by a senior attending neuroradiologist. Specific attention was given to the bilateral IONs on long-TR images. The IONs were evaluated for volume and signal changes independently. An abnormality was deemed to be HOD if it met the following criteria: 1) Both hypertrophy and signal changes (T2 prolongation) were present; 2) the hypertrophic component was modest or absent, but the signal changes were unequivocal. The findings within each ION for both T2- and proton attenuation-weighted sequences were classified as follows: $0=$ no visible abnormality, 1 = questionable abnormally high signal within the hilum or entire nucleus with or without volume changes, or $2=$ definite abnormally high signal within the hilum or entire nucleus with or without volume changes. A score of 2 at any point during the postoperative follow-up qualified the respective ION for HOD status. Patients were subsequently categorized as having definite bilateral HOD, definite unilateral HOD, or no HOD.

\section{Statistical Analysis}

Statistical analysis was performed by using SAS 9.2 software (SAS Institute, Cary, North Carolina). The Fisher exact test was performed to investigate the association of HOD-related MR imaging abnormalities with PFS. The sensitivity and specificity of HOD as an indicator of PFS were calculated by using the clinical diagnosis of PFS as the criterion standard (true-positive).

\section{RESULTS}

Of the 12 patients with clinically diagnosed PFS, 9 had bilateral HOD on MR imaging and 2 had definitive unilateral HOD changes ( 1 with questionable contralateral involvement), and 1 had no abnormal MR imaging findings that were consistent with HOD. Conversely, 10 of the 12 patients without PFS had no HOD-suggestive findings on MR imaging, and 2 had unilateral HOD. In cases of unilateral HOD, patients with PFS had HOD on the right side, and patients without PFS had it on the left. The earliest time when definite bilateral HOD was seen in our patients was 1 month from surgery, and the longest interval between surgery and the appearance of definite bilateral HOD was 5.5 months (mean, 3.5 months).

The clinical features of all 24 patients are summarized in the Table. All 12 patients with postoperative mutism had severe cerebellar syndrome with dysmetria and ataxia. All patients without PFS had some degree of cerebellar dysfunction without mutism, and 5 were quite severely affected. The 2 patients with clinical PFS but only unilateral HOD had mild postoperative dysmetria and 
Clinical features of patients with and without posterior fossa syndrome

\begin{tabular}{lcc}
\hline \multicolumn{1}{c}{ Variable } & PFS $(\boldsymbol{n}=12)$ & Non-PFS $(\boldsymbol{n}=12)$ \\
\hline Mutism $>$ 24 hours & 12 & 0 \\
Immediate postoperative & & 2 \\
$\quad$ Mild-to-moderate dysmetria & 7 & 0 \\
$\quad$ Severe dysmetria & 5 & 7 \\
Mild-to-moderate ataxia & 0 & 2 \\
$\quad$ Severe ataxia & 12 & \\
At last follow-up & & \\
Mild-to-moderate dysmetria & 2 & 0 \\
Severe dysmetria & 1 & 5 \\
$\quad$ Mild-to-moderate ataxia & 7 & 1 \\
$\quad$ Severe ataxia & 5 &
\end{tabular}

Note:-Dysmetria: incoordination of limbs with either some (i.e., mild to moderate) or complete (i.e., severe) impairment of function. Ataxia: impaired ability to sit and walk with independent mobility retained (mild to moderate) or with mobility assistance required (severe).

a Time to last follow-up evaluation: 3-99 months.

severe ataxia. The 2 patients who had unilateral HOD without clinical PFS had mild postoperative dysmetria and mild ataxia.

The fact that $100 \%$ of patients with bilateral HOD changes had PFS and $0 \%$ of patients without PFS had such changes suggests that the presence of bilateral HOD is associated with PFS. The results of the Fisher exact test confirmed the strong association between bilateral HOD and clinical PFS $(P<.0001)$. These findings also indicated a false-positive MR imaging rate of $0 \%(0 / 9)$ for bilateral HOD because all patients with bilateral HOD indeed had PFS. The false-negative MR imaging rate was 25\% (3/12). In this clinical setting, MR imaging findings of bilateral HOD were $75 \%$ sensitive and $100 \%$ specific for the diagnosis of PFS. With bilateral HOD as a putative indicator of PFS, the positive predictive value of bilateral HOD is $100 \%$ and the negative predictive value of the absence of HOD is $80 \%$.

\section{DISCUSSION}

As previously stated, damage to the bilateral pECPs is the most widely accepted cause of postoperative PFS and cerebellar mutism. ${ }^{5,8-12}$ Because of the anatomic overlap between the first segments of the dentatorubrothalamocortical pathway and the Guillain-Mollaret triangle, damage to the pECP leads to contralateral HOD. ${ }^{23-28}$ Therefore, damage to the bilateral pECPs should lead to the development of bilateral HOD on MR imaging, which may serve as a delayed surrogate imaging indicator of PFS. Indeed, the $100 \%$ positive predictive value yields a high confidence that a positive result (obtained by using bilateral HOD as a classifier) is truly indicative of PFS.

Although bilateral HOD has previously been associated with linguistic pathway abnormalities, ${ }^{29-32}$ we could not find any report of cerebellar mutism and linguistic abnormalities attributed to bilateral damage of the pECPs and with subsequent HOD in the ION. Most of the described cases in which unilateral HOD was observed on imaging did not have associated linguistic category findings, ${ }^{33-37}$ though this was described in at least 1 study. ${ }^{38}$

Bilateral HOD was not present in every case of clinically diagnosed PFS in our cohort. This divergence could be due to inconsistencies in the clinical diagnostic criteria because despite all ongoing effort and research, the diagnosis of PFS is sometimes still a judgment call, dependent on the investigator's experience. Our criteria of prolonged mutism and severe associated cerebellar syn- drome with ataxia were used to differentiate PFS from other causes of postoperative speech disorders, including damage to the lower cranial nerves, ${ }^{13}$ psychological issues, ${ }^{39}$ and medicationinduced deficits. Indeed, at least one of our patients with PFS without bilateral HOD on MR imaging was described in the medical records as having immediate mutism following anesthesia recovery, a presentation that is not typical of cerebellar mutism, which generally appears an average of 1.7 days after surgery. $3,5,40$ In fact, the immediate onset of mutism may indicate a bulbar abnormality from direct, inadvertent surgical injury to the lower cranial nerve nuclei ${ }^{13}$ rather than true cerebellar mutism in the context of PFS. We, therefore, recognize the challenges of differentiating cerebellar mutism from other forms of transient speech arrests in these patients during the postoperative period when other compounding clinical considerations occur concurrently.

Another reason that bilateral HOD was not always present in patients with PFS is that surgical damage is not necessarily a binary, all-or-none phenomenon. In other words, the magnitude of damage to the pECP that is needed to cause a critical drop of cerebellar input to the supratentorial brain may be different from the amount of damage leading the later to visible MR imaging changes of HOD. For example, one may hypothesize that most of the bilateral pECP fibers need to be destroyed to result in bilateral HOD, but somewhat less damage may be sufficient to cause a global frontal lobe dysfunction and resultant speech apraxia (ie, cerebellar mutism). Indeed, the 2 mechanisms causing PFS and HOD are likely different: one leading to frontal lobe dysfunction and mutism through reversed cerebellocerebral diaschisis; the other, to a peculiar form of degeneration associated with initial hypertrophy through a trans-synaptic mechanism with as yet poorly understood neurologic correlates.

Another unique clinical aspect of bilateral HOD in our patient cohort was the absence of palatal myoclonus in all patients, though this neurologic sign is commonly seen associated with HOD. The reason for this discrepancy is unclear. However, given that our cohort's lesions occurred in the specific circumstance of posterior fossa surgery and consistently in a specific location (ie, $\mathrm{pECP}$ ) but other lesions in the literature often involved injury to the second, descending (rubro-olivary) segment of the GuillainMollaret triangle, an unidentified anatomic explanation may exist, such as involvement of brain stem structures immediately adjacent to the descending tegmental tracts in the development of palatal myoclonus.

The MR imaging signs and evolution of HOD-type changes are well-described, with pathologic evaluation first revealing 6 distinct stages after destruction of the central tegmental tracts: no olivary changes (within 24 hours), olivary amiculum degeneration (typically 2-7 days), olivary hypertrophy (approximately 3 weeks), culminant olivary enlargement (approximately 8.5 months), olivary pseudohypertrophy (9.5 months), and olivary atrophy (after a few years). ${ }^{28}$ These findings were later classified into 3 stages on the basis of MR imaging of other cases and analysis of cases in the literature. ${ }^{27,34}$ The first is seen with T2 and proton attenuation signal hyperintensity without hypertrophy and may occur as early as 4 weeks after injury. The second stage demonstrates hypertrophy starting approximately 4 months after injury extending until hypertrophy resolves approximately 3-4 
years later. The third and final stage exhibits resolved hypertrophy with persistent high $\mathrm{T} 2$ and proton attenuation signal intensity and is noted to persist indefinitely. These concur with our observations.

We were fortunate that our retrospective analysis was performed on patients whose clinical imaging protocol had included both axial T2 and proton attenuation imaging. The proton attenuation sequence was often the most conclusive imaging sequence, especially on the earlier postoperative follow-up studies, as previously reported by others. ${ }^{41}$ However, our imaging protocol was not optimized to evaluate the ION, an area that is somewhat difficult to visualize on routine imaging and is often prone to artifacts. Our protocol was also limited by section thickness, which was routinely $5 \mathrm{~mm}$. Because the olivary body measures approximately $1.25 \mathrm{~cm}$, with the ION being in the bottom portion, only 1 or 2 sections could generally be obtained to evaluate the region of interest on traditional 5-mm imaging, with the possibility of significant partial volume averaging effects.

Our finding of unilateral HOD in patients with and without PFS may be due to the proposed process of 1 cerebellar hemisphere normally providing asymmetric input toward speech. There is no consensus in the literature as to which side provides more input, with an earlier article implicating the left cerebellar hemisphere ${ }^{42}$ and more recent articles implicating the right. ${ }^{43-47}$ In cases in which we detected unilateral HOD, patients with PFS had unilateral right HOD, and patients without PFS had unilateral left HOD. Other articles described inconclusive findings. ${ }^{38,48}$ Given the inconsistency between literature reports, it is possible that interindividual variations determine the dominant cerebellar hemisphere for speech and language production. Alternatively, complete damage to the dominant side may lead to PFS, even if damage to the subdominant pECP is partial, hence insufficient to cause corresponding HOD. This result would explain the few exceptions of clinical-imaging discrepancies (ie, clinical PFS syndrome in conjunction with unilateral HOD only).

Although bilateral HOD is not $100 \%$ sensitive for detecting PFS, these findings suggest that a threshold of damage to the pECPs is required for both MR imaging and clinical perceptibility. Future studies evaluating the degree of damage to the pECPs and defining PFS diagnostic criteria in a larger patient cohort with detailed clinical evaluation will be the next step in understanding this complex syndrome. Our data demonstrate that changes of HOD on MR imaging may be used in the future as an objective though a posteriori criterion in the diagnosis of PFS and may help clarify the definition of the clinical syndrome. Future evaluation will include prospective analysis of a larger cohort of patients with posterior fossa surgery who have PFS, with more robust diagnostic information (optimized imaging of the Guillain-Mollaret triangle and pECPs) and structured clinical information acquired and evaluated by experienced investigators to formulate standardized clinical and imaging diagnostic criteria for this complex, challenging syndrome.

\section{CONCLUSIONS}

Damage to the pECP manifests as contralateral HOD through disruption of the Guillain-Mollaret triangle and subsequent trans-synaptic degeneration. We provide objective imaging data demonstrating that damage to the bilateral pECPs manifests as bilateral HOD. Our false-positive MR imaging rate for bilateral HOD was $0 \%$; hence, bilateral HOD may be a delayed but reliable surrogate imaging marker for PFS. Therefore, MR imaging findings, especially those obtained by using imaging protocols that are optimized for the visualization of the ION, can be useful criteria in the diagnosis of PFS as a more robust definition is developed. Our findings contribute to the growing body of evidence supporting the role of bilateral pECP damage in the pathogenesis of postoperative PFS.

\section{ACKNOWLEDGMENTS}

The authors thank Cherise M. Guess, PhD, ELS, for reviewing and editing the manuscript.

Disclosures: Zoltan Patay_UNRELATED: Grants/Grants Pending: American Lebanese Syrian Associated Charities, ${ }^{*}$ National Cancer Institute, ${ }^{\star}$ Comments: The American Lebanese Syrian Associated Charities is the fundraising arm of St. Jude Children's Research Hospital; therefore, most research conducted at St. Jude is "funded" to some extent by the American Lebanese Syrian Associated Charities; St. Jude Children's Research Hospital is also a "Comprehensive Cancer Center" receiving a grant from the National Cancer Institute, Travel/Accommodations/Meeting Expenses Unrelated to Activities Listed: ERASMUS Course, European Course of Pediatric Neuroradiology, European Society of Neuroradiology, São Paulo Radiology Society, Kuwait Radiology Society, Hungarian Neuroradiology Society, Comments: travel and accommodation expense coverage for presenting educational and/or invited lectures at courses or annual scientific meetings (no honoraria). Jacob EnterkinUNRELATED: Travel/Accommodations/Meeting Expenses Unrelated to Activities Listed: Medical University of South Carolina, Comments: received reimbursement to attend the American Society of Spine Radiology Annual Symposium in February 2013 to present a paper. As a neuroradiology fellow, 1 meeting is reimbursed. Zoran Rumboldt_-UNRELATED: Grants/Grants Pending: Siemens, ${ }^{*}$ Comments: CT Perfusion for Head and Neck Cancer, Travel/Accommodations/Meeting Expenses Unrelated to Activities Listed: Siemens, Bracco. Frederick Boop—UNRELATED: Board Membership: Secretary, American Association of Neurological Surgeons.* *Money paid to the institution.

\section{REFERENCES}

1. Hirsch JF, Renier D, Czernichow P, et al. Medulloblastoma in childhood: survival and functional results. Acta Neurochir (Wien) 1979;48:1-15

2. Stein BM, Fraser RA, Tenner MS. Normal pressure hydrocephalus: complication of posterior fossa surgery in children. Pediatrics 1972;49:50-58

3. Gudrunardottir T, Sehested A, Juhler M, et al. Cerebellar mutism: review of the literature. Childs Nerv Syst 2011;27:355-63

4. Gudrunardottir T, Sehested A, Juhler M, et al. Cerebellar mutism: definitions, classification and grading of symptoms. Childs Nerv Syst 2011;27:1361-63

5. Erşahin Y, Mutluer S, Cağli S, et al. Cerebellar mutism: report of seven cases and review of the literature. Neurosurgery 1996;38:6065, discussion 66

6. Pollack IF, Polinko P, Albright AL, et al. Mutism and pseudobulbar symptoms after resection of posterior fossa tumors in children: incidence and pathophysiology. Neurosurgery 1995;37:885-93

7. Riva D, Giorgi C. The cerebellum contributes to higher functions during development: evidence from a series of children surgically treated for posterior fossa tumours. Brain 2000;123(pt 5):1051-61

8. Morris EB, Phillips NS, Laningham FH, et al. Proximal dentatothalamocortical tract involvement in posterior fossa syndrome. Brain 2009;132:3087-95

9. Miller NG, Reddick WE, Kocak M, et al. Cerebellocerebral diaschisis is the likely mechanism of postsurgical posterior fossa syndrome in pediatric patients with midline cerebellar tumors. AJNR Am J Neuroradiol 2010;31:288-94

10. Puget $S$, Boddaert N, Viguier D, et al. Injuries to inferior vermis and dentate nuclei predict poor neurological and neuropsychological 
outcome in children with malignant posterior fossa tumors. Cancer 2009; $115: 1338-47$

11. Crutchfield JS, Sawaya R, Meyers CA, et al. Postoperative mutism in neurosurgery: report of two cases. J Neurosurg 1994;81:115-21

12. Koh S, Turkel SB, Baram TZ. Cerebellar mutism in children: report of six cases and potential mechanisms. Pediatr Neurol 1997; 16:218-19

13. Robertson PL, Muraszko KM, Holmes EJ, et al. Incidence and severity of postoperative cerebellar mutism syndrome in children with medulloblastoma: a prospective study by the Children's Oncology Group. J Neurosurg 2006;105:444-51

14. Fraioli B, Guidetti. Effects of stereotactic lesions of the dentate nucleus of the cerebellum in man. Appl Neurophysiol 1975;38:81-90

15. Steinbok P, Cochrane DD, Perrin R, et al. Mutism after posterior fossa tumour resection in children: incomplete recovery on longterm follow-up. Pediatr Neurosurg 2003;39:179-83

16. Kusano $Y$, Tanaka $Y$, Takasuna $H$, et al. Transient cerebellar mutism caused by bilateral damage to the dentate nuclei after the second posterior fossa surgery: case report. J Neurosurg 2006;104:329-31

17. Rekate HL, Grubb RL, Aram DM, et al. Muteness of cerebellar origin. Arch Neurol 1985;42:697-98

18. Pollack IF. Posterior fossa syndrome. Int Rev Neurobiol 1997;41:411-32

19. Devinsky O, Morrell MJ, Vogt BA. Contributions of anterior cingulate cortex to behaviour. Brain 1995;118(pt 1):279-306

20. Nagaratnam N, Nagaratnam K, Ng K, et al. Akinetic mutism following stroke. J Clin Neurosci 2004;11:25-30

21. Tahta K, Cirak B, Pakdemirli E, et al. Postoperative mutism after removal of an anterior falcine meningioma. J Clin Neurosci 2007;14:793-96

22. Guillain G, Mollaret P. Deux cas de myoclonies synchrones et rythmées vélo-pharyngo-laryngo-oculo-diaphragmatiques. Rev Neurol 1931;12:545-66

23. Gautier JC, Blackwood W. Enlargement of the inferior olivary nucleus in association with lesions of the central tegmental tract or dentate nucleus. Brain 1961;84:341-61

24. Jellinger K. Hypertrophy of the inferior olives. Report on 29 cases. $Z$ Neurol 1973;205:153-74

25. Krings T, Foltys H, Meister IG, et al. Hypertrophic olivary degeneration following pontine haemorrhage: hypertensive crisis or cavernous haemangioma bleeding? J Neurol Neurosurg Psychiatry 2003;74:797-99

26. Uchino $A$, Hasuo $K$, Uchida $K$, et al. Olivary degeneration after cerebellar or brain stem haemorrhage: MRI. Neuroradiology 1993;35:335-38

27. Goyal M, Versnick E, Tuite P, et al. Hypertrophic olivary degeneration: metaanalysis of the temporal evolution of MR findings. AJNR Am J Neuroradiol 2000;21:1073-77

28. Goto N, Kaneko M. Olivary enlargement: chronological and morphometric analyses. Acta Neuropathol 1981;54:275-82

29. Gerace C, Fele MR, Luna R, et al. Neurological picture: bilateral hypertrophic olivary degeneration. J Neurol Neurosurg Psychiatry 2006;77:73
30. Rieder CR, Reboucas RG, Ferreira MP. Holmes tremor in association with bilateral hypertrophic olivary degeneration and palatal tremor: chronological considerations-case report. Arq Neuropsiquiatr 2003;61:473-77

31. Vaidhyanath R, Thomas A, Messios N. Bilateral hypertrophic olivary degeneration following surgical resection of a posterior fossa epidermoid cyst. Br J Radiol 2010;83:e211-215

32. Shepherd GM, Tauboll E, Bakke SJ, et al. Midbrain tremor and hypertrophic olivary degeneration after pontine hemorrhage. Mov Disord 1997;12:432-37

33. Akar S, Drappatz J, Hsu L, et al. Hypertrophic olivary degeneration after resection of a cerebellar tumor. J Neurooncol 2008;87:341-45

34. Birbamer G, Buchberger W, Kampfl A, et al. Early detection of posttraumatic olivary hypertrophy by MRI. J Neurol 1993;240:407-09

35. Macht S, Hänggi D, Turowski B. Hypertrophic olivary degeneration following pontine cavernoma hemorrhage: a typical change accompanying lesions in the Guillain-Mollaret triangle. Klin Neuroradiol 2009;19:235-37

36. Phatouros CC, McConachie NS. Hypertrophic olivary degeneration: case report in a child. Pediatr Radiol 1998;28:830-31

37. Salamon-Murayama N, Russell EJ, Rabin BM. Diagnosis please: case 17-hypertrophic olivary degeneration secondary to pontine hemorrhage. Radiology 1999;213:814-17

38. Ash L, Srinivasan A. Case of the season: hypertrophic olivary degeneration. Semin Roentgenol 2008;43:171-72

39. Ferrante L, Mastronardi L, Acqui M, et al. Mutism after posterior fossa surgery in children: report of three cases. J Neurosurg 1990;72:959-63

40. Gelabert-González M, Fernández-Villa J. Mutism after posterior fossa surgery: review of the literature. Clin Neurol Neurosurg 2001;103:111-14

41. Yokota T, Hirashima F, Furukawa T, et al. MRI findings of inferior olives in palatal myoclonus. J Neurol 1989;236:115-16

42. Lechtenberg R, Gilman S. Speech disorders in cerebellar disease Ann Neurol 1978;3:285-90

43. Gebhart AL, Petersen SE, Thach WT. Role of the posterolateral cerebellum in language. Ann N Y Acad Sci 2002;978:318-33

44. Stoodley CJ, Schmahmann JD. Functional topography in the human cerebellum: a meta-analysis of neuroimaging studies. Neuroimage 2009;44:489-501

45. Baillieux H, De Smet HJ, Paquier PF, et al. Cerebellar neurocognition: insights into the bottom of the brain. $\mathrm{Clin} \mathrm{Neu}$ rol Neurosurg 2008;110:763-73

46. Ackermann H, Mathiak K, Ivry RB. Temporal organization of "internal speech" as a basis for cerebellar modulation of cognitive functions. Behav Cogn Neurosci Rev 2004;3:14-22

47. Fiez JA, Petersen SE, Cheney MK, et al. Impaired non-motor learning and error detection associated with cerebellar damage: a single case study. Brain 1992;11(pt 1):155-78

48. Bruno MK, Wooten GF. Hypertrophic olivary degeneration. Arch Neurol 2012;69:274-75 